\title{
Beyond control: Applying ecological research to improve long-term outcomes of wilding conifer management.
}

Ian Dickie ${ }^{1}$, Rowan Sprague ${ }^{2}$, Duane A Peltzer ${ }^{3}$, Joanna Green ${ }^{4}$, Kate Orwin ${ }^{3}$, and Sarah Sapsford ${ }^{4}$

${ }^{1}$ BioProtection Research Centre, School of Biological 8 Sciences, Te Kura Pūtaiao Koiora, Te Whare Wānanga o Waitaha | University of Canterbury

${ }^{2}$ New Zealand Wilding Conifer Group, Christchurch, New Zealand

${ }^{3}$ Manaaki Whenua Landcare Research, Lincoln, New Zealand

${ }^{4}$ BioProtection Research Centre, School of Biological Sciences, University of Canterbury, New Zealand

January 24, 2022

\section{Hosted file}

Beyond control_7.pdf available at https://authorea.com/users/368780/articles/526946-beyondcontrol-applying-ecological-research-to-improve-long-term-outcomes-of-wilding-conifermanagement 